\title{
Vaginal stenosis with cervical intraepithelial neoplasia in a postmenopausal patient with pyometra: a diagnostic dilemma
}

\author{
Jenifer Ahmad*, Meena N. Satia, V. R. Badhwar
}

Department of Obstetrics and Gynecology, Dr. D. Y. Patil Medical College, Navi Mumbai, Maharashtra, India

Received: 11 November 2019

Revised: 23 December 2019

Accepted: 26 December 2019

\author{
*Correspondence: \\ Dr. Jenifer Ahmad, \\ E-mail: jeniferahmadsheran@icloud.com
}

Copyright: ( $)$ the author(s), publisher and licensee Medip Academy. This is an open-access article distributed under the terms of the Creative Commons Attribution Non-Commercial License, which permits unrestricted non-commercial use, distribution, and reproduction in any medium, provided the original work is properly cited.

\begin{abstract}
Postmenopausal patient presenting with pain and lump in abdomen should be considered as a complication caused by gynaecological malignancy unless proved otherwise pyometra is most common in postmenopausal women but it can affect women of child bearing age as well. Usually it is caused by impairment of natural drainage of the cervix due to malignant diseases of genital tract and consequences of their treatment with radiotherapy. Authors report one such case with cervical intraepithelial neoplasia with uterus markedly enlarged to 26-28 weeks size with Vaginal stenosis in an eighty-year-old postmenopausal patient.
\end{abstract}

Keywords: Cervical intraepithelial neoplasia, Malignancy, Postmenopausal, Pyometra, Stenosis, Vaginal

\section{INTRODUCTION}

Cervical intraepithelial neoplasia occurring at the transitional zone between squamous epithelium of the vagina and columnar epithelium of endocervix is very rarely encountered in the eighth decade of life. These lesions usually occur around the age group of 30-40 years of age and a decade later likely to progress into invasive lesion. Authors report a rare case of CIN resulting in pyometra and vaginal stenosis. Pyometra is a rare condition characterized by the accumulation of pus within the uterine cavity with an incidence of $0.01-0.5 \%$ of female patients. ${ }^{1}$ Most commonly these could be due to endometrial or cervical malignancy associated with impairment of natural drainage due to cervical or vaginal stenosis or due to complications of radiotherapy. The incidence of pyometra increases with age and is $13.6 \%$ in elderly patients. Median age of presentation is 65 years and less than one-third are associated with underlying malignancy. $^{2}$ the main complications are bacteremia, sepsis and spontaneous uterine perforation with generalized peritonitis. Other less common causes include senile vaginitis or cervicitis, endometrial polyps puerperal infections, genital tuberculosis, cervical occlusion after surgery, leiomyoma and, congenital cervical anomalies and forgotten intra-uterine devices. ${ }^{3}$ Premalignant lesions of the cervix with super added infection may lead to agglutination of anterior and posterior vaginal walls resulting in vaginal stenosis as was seen in our patient. Pyometra in these patients hardly respond to antibiotics.

\section{CASE REPORT}

Eighty-year-old P6 post-menopausl for 25 years patient presented with pain in abdomen with abdominal distension since one month with mass in abdomen which had increased in size in the last one month. she also complained of dyspepsia and bloating sensation since last 8-10 days. On clinical examination vital parameters were stable. On abdominal examination a mobile mass of 2628 weeks size arising from pelvis firm in consistency 
with tenderness was palpated. On vaginal examination cervix was not felt only lower one third of vagina was felt with bulging anterior and posterior fornices with no free space between the cystic mass and lateral pelvic walls and a clinical impression of inoperable ovarian tumor or Pyometra was made and sonography pelvis along with tumor markers was advised.

Ultrasonography was suggestive of Rt ovarian mass $17 \times$ $7.6 \mathrm{~cm}$ bilobed heterogeneously hypoechoic lesion with echoes with in-it.

Her tumor markers were all within normal limits $\mathrm{Ca} 125$ 12 IU\ml CEA-3.2 ng/ml Ca 19.9-15.5 IU/ml LDH-575 IU/L In view of negative tumor marker a CT scan was advised and the report was suggestive of bilobed thick walled hypodense cystic lesion collection arising from the pelvis extending into the abdomen up to the supraumbilical region with few foci of peripheral calcification and multiple dense moving internal echoes suggestive of haemetro/pyometra with pyocolpus with both ovaries normal and separate from the lesion as shown in the following Figures.

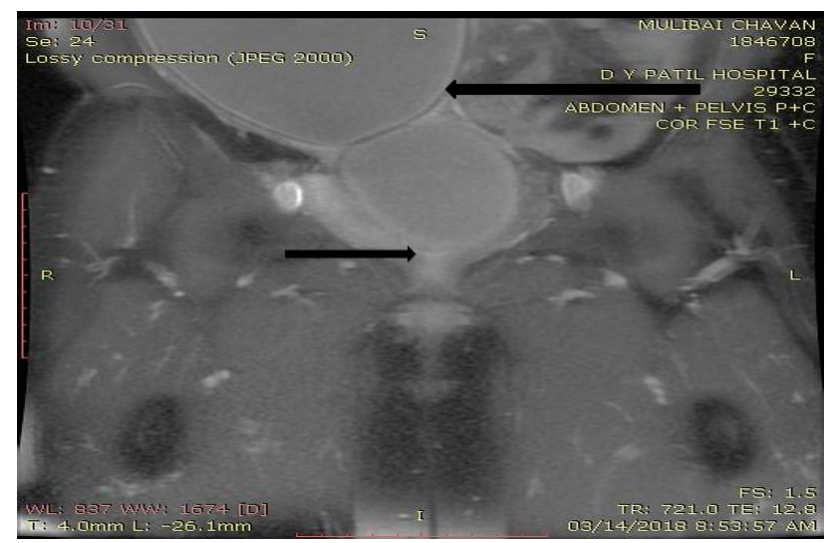

CT scan abdo + pelvis - (arrow on right) represent cystic lesion arising from pelvis extending into abdomen up to supraumblical region with few foci of peripheral calcification. (Arrow on left) represent the lobes of cyst along with the collection with in.

\section{Figure 1: CT scan plain + contrast images of} abdomen and pelvis.

In view of the above finding patient was posted for EUA and if required total abdominal hystrectomy along with bilateral salpingo-opherectomy. Intraoperative findings after spinal anesthesia a gentle per vaginal examination was tried during which a gush of brownish coloured fluid was seen from vagina around 2 litres of fluid was drained after which the mass of 26-28 weeks size subsided and on repeated pelvic examination cervix was fond to be normal but stenosed. Bilateral fornix were clear and uterus was soft flabby and bulky immediately decision for hysterectomy was taken as pyometra could be most commonly due to endometrial or cervical malignancy associated with impairment of drainage due to cervical stenosis.

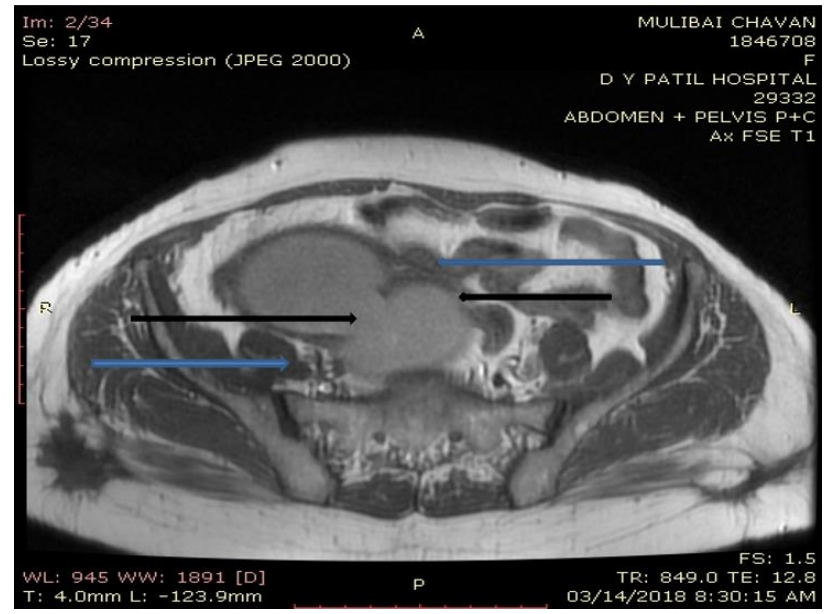

(Arrow on right) connection between the lobes of cyst. (Arrow on left) bilobed thick walled hypodense cystic lesion collection arising from the pelvis extending into the abdomen with multiple dense moving internal echoes. (Pointed with blue arrow) both ovaries normal and separate from the lesion.

Figure 2: CT scan abdo + pelvis.

Intra-op findings as shown in Figure 3 and 4.

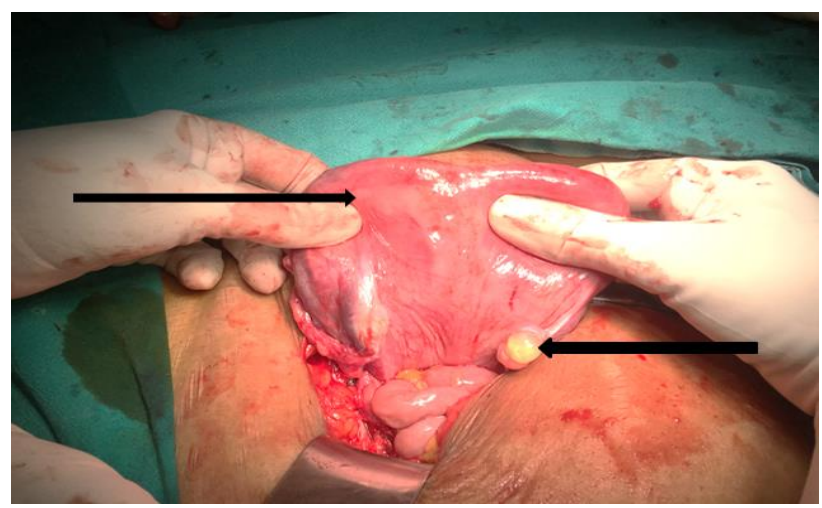

(Right arrow) - flabby uterus measuring $8 \times 10 \mathrm{~cm}$. (left arrow) - ovary of left side.

Figure 3: Intra-op uterus.

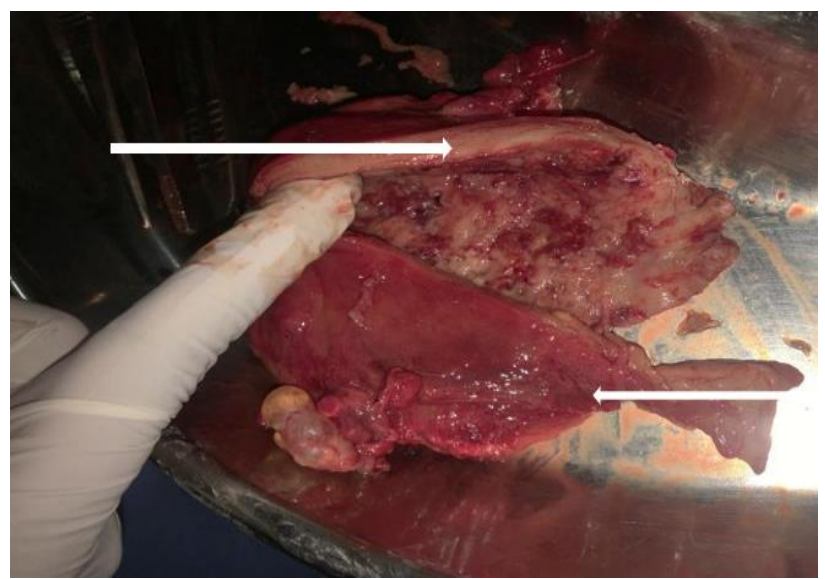

(Right arrow) thin walled and flabby uterus, (left arrow) stenosed and atrophied cervix.

Figure 4: Cut section of uterus. 
Total abdominal hystrectomy along with bilateral salphingo-ophorectomy was done and the specimen was sent for histopath examination.

\section{Histopath report}

Suggestive of high grade squamous intraepithelial lesion of cervix as shown in Figure 4 uterus was suggestive of pyometra with simple serous cyst of right ovary.

Histopathology report showing CIN.

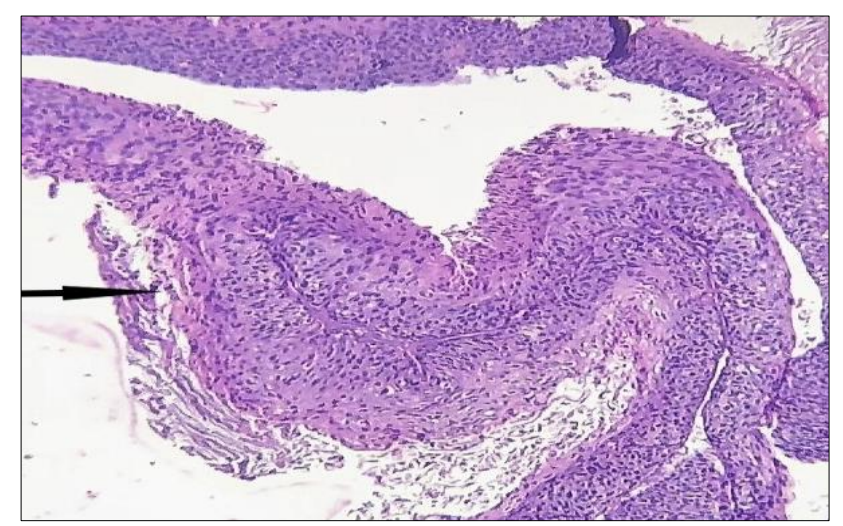

High grade squamous intraepithelial neoplasia of cervix.

Figure 5: Histopath slide of cervix.

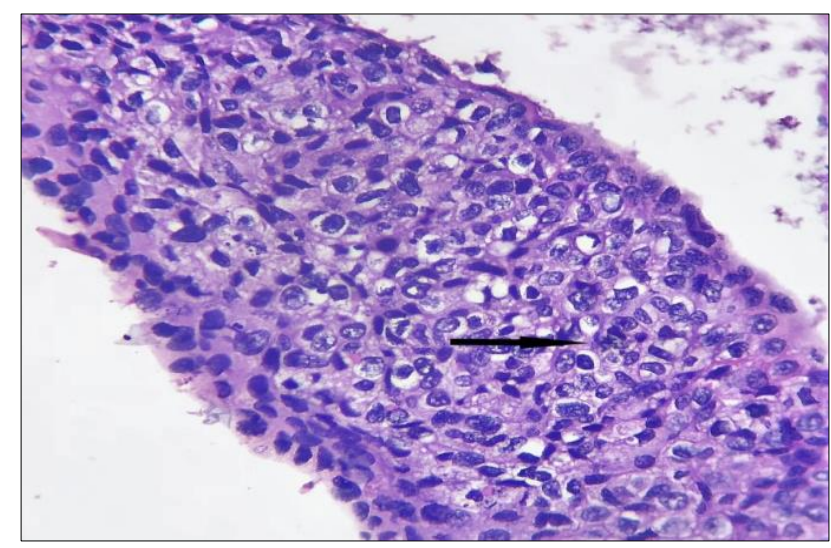

Figure 6: Histopath slide of cervix.

Magnified image of same slide as shown in pointed with arrow in Figure 5. Showing neoplastic cells suggestive of high grade of squamous intraepithelial neoplasia of cervix (Figure 6).

\section{DISCUSSION}

During menopause the cervical and vaginal tissues may become thin and atrophic leading pale pink vagina with loss of rugosity and cervical and vaginal stenosis malignancies of the cervix or the endometrium or conservative surgeries done on premalignant lesions of the cervix may result in vaginal stenosis Procedures that destroy or remove the lining of the uterus for example endometrial ablation in women who have persistent vaginal bleeding and as a complication of radiation therapy to treat cervical cancer or endometrial cancer also maybe the cause of stenosis This may result in an accumulation of blood in the uterus leading to hematometra. Various studies have shown that $75 \%$ of patients who presented with pyometra due to cervical and vaginal stenosis had gynaecological malignancy had endometrial cancer in $41.6 \%$, cervical cancer in $25 \%$, liomyosarcoma in $8.3 \%$ of cases. In $25 \%$ of patients the cause of pyometra was benign pathologies like vaginal atrophy. ${ }^{4}$

Rarely in some women in the child bearing age group retrograde flow into the pelvis may lead to endometriosis. Pus may accumulate in the uterus as it does in some women with cervical or endometrial cancer leading to pyometra Women with vaginal stenosis may present with a short or narrowed vagina, which may make vaginal intercourse and even pelvic examination painful or impossible..$^{5}$ Dryness and itching may be the symptoms associated with Vaginal stenosis. The use of HRT in some patients may assist in epithelial regeneration, local application of benzydamine may relieve symptoms of acute radiation vaginitis. Vaginal dilators are used to prevent and treat vaginal stenosis only after ruling out malingnancy. Sometimes surgical interventions may be required to treat vaginal stenosis. However, it is important to note that skin in an irradiated field is less likely to heal, so other measures should be tried before considering surgical treatment. Those, not associated with malignancy have better prognosis as compared to those cases that are associated with malignancy. ${ }^{6}$ In patients with cervical or endometrial cancers radical hysterectomy with or without radiotherapy would be recommended.

\section{CONCLUSION}

Pyometra is a dangerous medical condition not only because of its association with malignancy but also there is a significant risk of septicaemia. Prompt diagnosis in postmenopausal women presenting with abdominal distension early intervention, intensive pre- and postoperative care, and proper treatment can reduce morbidity and mortality. With the proper clinician support and teaching, vaginal stenosis at times can be diagnosed and controlled with invasive/non-invasive treatment modalities.

\section{Funding: No funding sources \\ Conflict of interest: None declared \\ Ethical approval: Not required}

\section{REFERENCES}

1. Lee DH, Cho DH, Lee JH, Kim YR. A case of postmenopausal pyometra caused by endometrial tuberculosis. Korean J Obstet Gynecol. 2012;55:42932.

2. Chauhan SCA, Sherama LCMM, Banerjee BJK. Spontaneous perforation of pyometra: a rare cause of 
diffuse peritonitis. Med J Armed Forces India. 2015;71:192-3.

3. Yadav P, Poddar DP, Chavan K, Saraogi R. Huge pyometra in a postmenopausal age: a diagnostic dilemma. Int J Reprod Contracept Obstet Gynecol. 2015;4:1549-51.

4. Wolf, Judith. Prevention and treatment of vaginal stenosis resulting from pelvic radiation therapy. Community Oncol. 2006;3:10.

5. Kerimoglu OS, Pekin A, Yilmaz SA, Bakbak BB, Celik C. Pyometra in elderly post-menopausal women: a sign of malignity. Eur J Gynaecol Oncol. 2015;36(1):59-61.

6. Inui A, Nitta A, Yamamoto A. Generalized peritonitis with pneumoperitoneum caused by the spontaneous perforation of pyometra without malignancy: Report of a case. Surg Today. 1999;29:935.

Cite this article as: Ahmad J, Satia MN, Badhwar VR. Vaginal stenosis with cervical intraepithelial neoplasia in a postmenopausal patient with pyometra: a diagnostic dilemma. Int J Reprod Contracept Obstet Gynecol 2020;9:842-5. 